\title{
Forensic luminol reaction for detecting fecal occult blood in experimental mice
}

\author{
Ah-Mee Park*,1 \& Ikuo Tsunoda ${ }^{1}$ \\ ${ }^{1}$ Department of Microbiology, Kindai University Faculty of Medicine, Osakasayama, Osaka 589-8511, Japan
}

BioTechniques 65: 227-230 (October 2018) 10.2144/btn-2018-0017

Keywords: animals $\bullet$ gastrointestinal hemorrhage $\bullet$ laboratory $\bullet$ luminescence $\bullet$ stool

Fecal occult blood (FOB) is a sign of gastrointestinal diseases, such as intestinal ulcers and colorectal cancer. In experimental animal studies, there is no standard method to detect FOB. Here, we present a simple protocol to detect FOB in mice, using the Luminol Reaction Experiment $\mathrm{Kit}^{\circledR}$ that was originally designed to detect bloodstains at a crime scene in criminal forensics. To obtain positive control bloody feces, we used an indomethacin-induced intestinal ulcer model in mice. By mixing small pieces of feces with a luminol solution, the fecal solution emitted visible blue-white chemiluminescence in dark field when feces contained hemoglobin. We also established a method for semi-quantification of hemoglobin content in the fecal solution, using a luminometer. This method is simple, quick, economical and semi-quantitative, allowing researchers to detect FOB in experimental mice.

Fecal occult blood (FOB) is a sign of gastrointestinal diseases, including ulcers [1] and colorectal cancer [2-4]. Clinically, there are two standard methods to detect FOB: the stool guaiac test is based on the oxidation of guaiac by hydrogen peroxide $\left(\mathrm{H}_{2} \mathrm{O}_{2}\right)$, which is catalyzed by the peroxidase-like activity of hemoglobin $(\mathrm{Hb})$ [5]; while the immunochemical test using anti-human $\mathrm{Hb}$ antibodies is more specific and expensive than the guaiac test [2]. For experimental animal studies, however, there is no standard method to detect FOB. Here, we have established a simple method to detect FOB in experimental mice, using the Luminol Reaction Experiment Kit ${ }^{\circledR}$ (Wako Pure Chemical, Osaka, Japan), which has been used in criminal forensics to detect blood at a crime scene. The luminol test is based on the oxidation of the substrate by the peroxidase-like activity of $\mathrm{Hb}$, where the luminol reagents emit visible blue-white chemiluminescence in dark field.

As a positive control, we used a nonsteroidal anti-inflammatory druginduced intestinal ulcer model in mice [6], where FOB is detectable $4 \mathrm{~h}$ after intragastric indomethacin administration. The animal experiments were approved by the Institutional Animal Care and Use Committee of Kindai University and performed in accordance with the institutional guidelines. We harvested feces from CD1 mice (Charles River Laboratories Japan, Yokohama, Japan) at different time points following indomethacin administration (6 mg/kg body weight). The luminol solution was prepared by mixing two reagents (luminol and sodium peroxide) in deionized-distilled water $\left(\mathrm{ddH}_{2} \mathrm{O}\right)$, according to the manufacturer's instruction. Because of the high sensitivity of the luminol solution, we used a dilute solution (fivefold with $\mathrm{dd}_{2} \mathrm{O}$ ) as a 'working solution' for visual detection.

Following addition of the working solution to FOB-positive feces, luminol generated blue-white luminescence (Figure 1A, Supplementary video 1). We also conducted a time-course analysis of FOB following indomethacin administration (Figure 1B). We placed a portion of feces $(<1 \mathrm{mg})$ in a 96-well plate (Thermo Fisher Scientific, MA, USA) at different time points and added $100 \mu$ l of the working solution into each well. Blue-white chemiluminescence became detectable at $4 \mathrm{~h}$ after the indomethacin administration and luminescence intensity increased thereafter.
To apply the luminol reaction for estimation of $\mathrm{Hb}$ concentrations in feces, we determined the range of fecal sample weights applicable for the luminol reaction. We prepared a FOB-positive fecal solution ( $1 \mathrm{mg} / \mathrm{ml}$ ) with $\mathrm{ddH}_{2} \mathrm{O}$, centrifuged it at $8000 \times \mathrm{g}$ for $2 \mathrm{~min}$, and the supernatant was used for assay. We placed 1-10 $\mu$ of the solution in 96-well plates, added $100 \mu$ l of the 500-fold dilute luminol solution, and measured its chemiluminescence signals using a microplate reader (ARVO; Perkin Elmer, MA, USA). In the range of 1 to $10 \mu \mathrm{g}$ feces/reaction, we obtained excellent linearity between chemiluminescence signals and fecal amounts (Figure 1C), while fecal amounts higher than $40 \mu \mathrm{g}$ decreased chemiluminescence signals (data not shown). Although the absolute chemiluminescence signal of the luminol solution differed in each assay, the ratio to the background chemiluminescence signal was stable (data not shown). Thus, we can obtain reproducible results by using the ratio to the background chemiluminescence (blank wells containing the luminol solution alone).

To determine whether the chemiluminescence signals could reflect the results of the above visual detection method,

By mixing bloody feces with a luminol solution, the solution emits chemiluminescence. The chemiluminescence is not only seen by the naked eye in dark field but is also measurable by a luminometer for semi-quantification. 
(A)

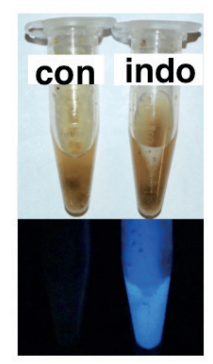

(C)

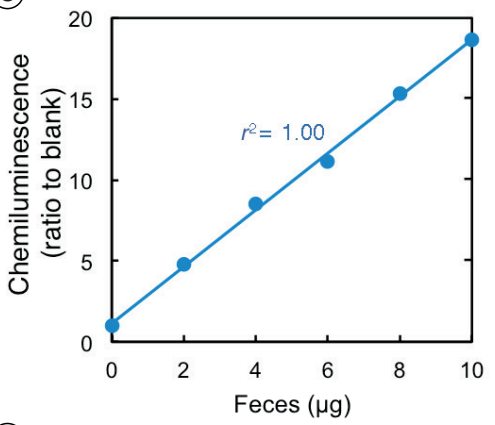

(E)

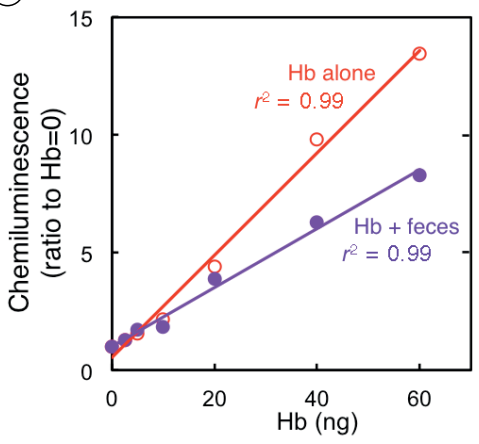

(B)

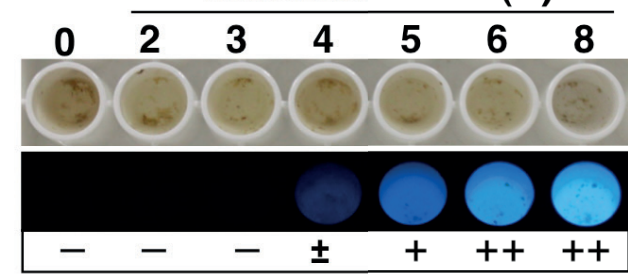

(D)

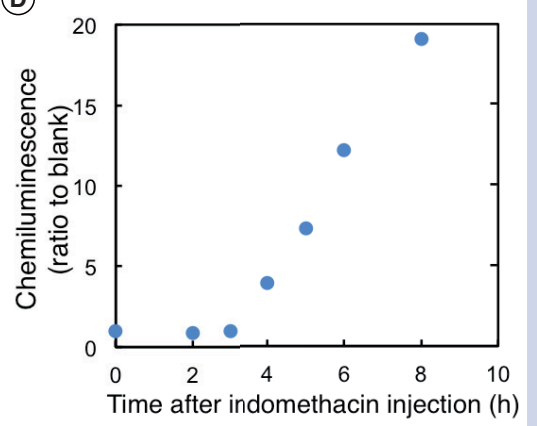

(

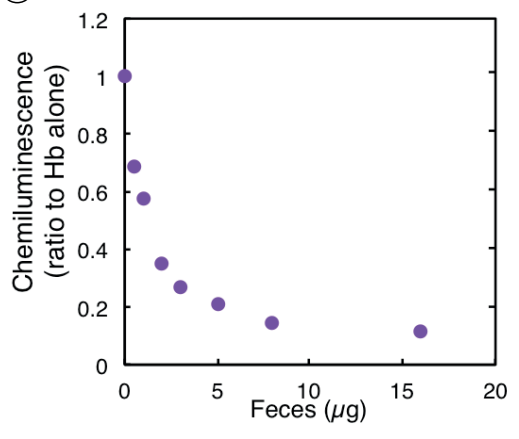

Figure 1. Fecal occult blood (FOB) detection by the luminol reaction. (A \& B) We induced intestinal ulcer in mice injected with indomethacin, and harvested feces. (A) Feces in a microtube at 0 (con) and 6 hours (h) (indo) and (B) a portion of feces in a 96-well plate at different time points $(0,2,3,4,5,6$ and 8 h) following the indomethacin injection. Fecal samples were mixed with a luminol solution and observed in dark field. The blue-white chemiluminescence became detectable at $4 \mathrm{~h}$ after the injection: -: negative; \pm : dim; +: moderate; and ++: bright. (C) Different amounts of fecal samples from A were placed into a 96 -well plate and mixed with $100 \mu \mathrm{l}$ of a dilute luminol solution. Chemiluminescence intensities were measured by a microplate reader. We calculated the ratios of the chemiluminescence intensities of wells containing different amounts of feces to the chemiluminescence intensity of wells containing a luminol solution alone. Weights of feces containing occult blood positively correlated with the chemiluminescence ratios $\left(r^{2}=1.00\right)$. (D) We placed $5 \mu \mathrm{g}$ of fecal samples from $\mathrm{B}$, mixed with the luminol solution, and calculated the chemiluminescence ratios to the chemiluminescence intensity of wells containing feces from untreated mouse (time: $0 \mathrm{~h}$ ). (E) We placed different amounts of $\mathrm{Hb}$ in 96 well plates in the absence ('Hb alone', open red circles) or the presence of ('Hb + feces', closed purple circles) $5 \mu \mathrm{g}$ of control feces, followed by addition of the luminol solution. The chemiluminescence intensity of the luminol solution alone or with $5 \mu \mathrm{g}$ of feces was used for the chemiluminescence ratios of ' $\mathrm{Hb}$ alone' or ' $\mathrm{Hb}+$ feces', respectively. Both $\mathrm{Hb}$ concentrations of 'Hb alone' or 'Hb + feces' samples positively correlated with the chemiluminescence ratios, although the ratios of the latter samples showed lower chemiluminescence ratios. (F) We placed different amounts of feces from normal mice (1-16 $\mu \mathrm{g} /$ well) into wells containing $50 \mathrm{ng}$ of $\mathrm{Hb}$, followed by addition of the luminol solution. We calculated the chemiluminescence ratios to the chemiluminescence intensity of wells containing $50 \mathrm{ng}$ of $\mathrm{Hb}$ without feces. (A-F) We repeated each experiment at least three times.

Con: Control; Hb: Hemoglobin; Indo: Indomethacin.

we used the same samples of Figure 1B and detected FOB by the chemiluminescence signals. We prepared fecal sample solutions $(1 \mathrm{mg} / \mathrm{ml})$ at different time points, placed $5 \mu \mathrm{g}$ of feces in 96-well plates, and measured their chemiluminescence signals. The signals became detectable $4 \mathrm{~h}$ after indomethacin administration and increased thereafter (Figure 1D). Thus, the chemiluminescence results were similar to the results observed by the visual detection.
Next, we quantified the Hb concentrations in feces, using $\mathrm{Hb}$ standard solutions derived from normal murine blood ( $\mathrm{Hb}$ concentration was determined by Hematology Analyzer XT-1800; Sysmex Corporation, Hyogo, Japan). In the range of 1-60 ng/well, $\mathrm{Hb}$ concentration curves showed excellent linearity with chemiluminescence ratios (Figure 1E); the detection limit was $1 \mathrm{ng} \mathrm{Hb} /$ well, while Hb concentrations higher than $60 \mathrm{ng} /$ well reached a plateau (data not shown).

Since feces themselves have been shown to affect the chemiluminescence [7], we measured the chemiluminescence signals of wells containing $50 \mathrm{ng}$ of $\mathrm{Hb}$ in the presence of different amounts of feces from normal mice (0-16 $\mu$ g of feces/ well). The addition of feces decreased the signals; in the presence of $5 \mu \mathrm{g}$ of feces, the signal decreased to $20 \%$ of that of a control well containing $50 \mathrm{ng}$ of $\mathrm{Hb}$ alone (Figure 1F). The signals in the presence of $5 \mu \mathrm{g}$ of feces/well provided the most reproducible results among different fecal amounts tested.

Lastly, we attempted to estimate the $\mathrm{Hb}$ concentrations in feces. We calculated the chemiluminescence ratios of wells containing different amounts of $\mathrm{Hb}$ in the presence of $5 \mu \mathrm{g}$ of feces from control mice (Figure 1E). Although the presence of feces decreased the ratios (and signals, data not shown) compared with those of wells containing no feces ('Hb alone'), their $\mathrm{Hb}$ concentrations curves showed excellent linearity with chemiluminescence ratios $\left(r^{2}=0.99\right)$.

We have established two simple protocols to detect FOB by visual observation or chemiluminescence measurement (Figure 2). We propose a scoring system for $\mathrm{FOB}$, which can be used in either protocol. For practical use, there are three tips for obtaining reproducible results as follows: first, the absolute value of chemiluminescence of the luminol solution can differ in each experiment; one needs to either set up wells containing $5 \mu \mathrm{g}$ of a control fecal sample from normal mice to calculate the chemiluminescence ratios, or freeze and store all fecal samples so that all samples can be analyzed simultaneously in a single experiment. Second, the chemiluminescence decreases rapidly once the luminol reaction is initiated (Supplementary video 2); the chemiluminescence needs to be measured immediately following addition of the luminol solution. Third, several 


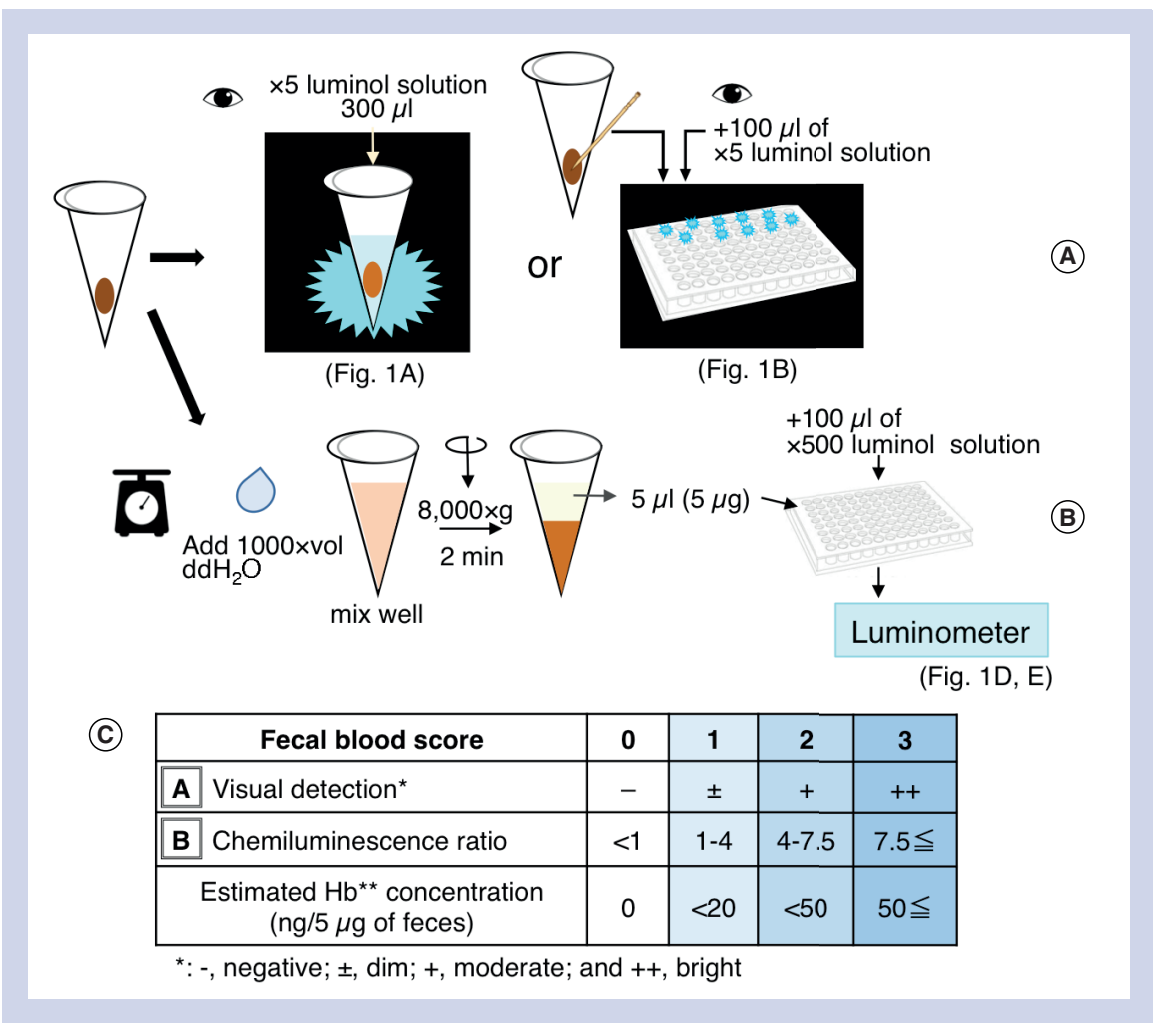

compounds affect the luminol reaction [8]. In our study, contamination with low levels of phosphate-buffered saline (PBS) and murine urine decreased the luminol reaction, while contamination with bleach (sodium hypochlorite, Wako Pure Chemical) and $\mathrm{H}_{2} \mathrm{O}_{2}$ increased the reaction: $50 \%$ and 99\% decreases by $1 \mu$ of PBS and normal urine/100 $\mu$ reaction, respectively; sixfold and fourfold increases by $1 \mu$ of 100 -times diluted sodium hypochlorite and $1 \mu$ of $3 \%$ $\mathrm{H}_{2} \mathrm{O}_{2}$ solution/100 $\mu$ reaction, respectively. In summary, our FOB detection method is simple, quick, economical and semi-quantitative, allowing researchers to detect FOB in experimental mice. It would be interesting to compare this method to other methods, such as the guaiac test and the immunochemical test in the future.

\section{Author contributions}

Study concept and design: A-MP and IT; experiments: A-MP; drafting the manuscript: A-MP and IT; obtaining fundings: A-MP and IT.

\section{Financial \& competing interests disclosure}

This work was supported by grants from the National Institute of General Medical Sciences COBRE Grant (P30-GM110703,
I Tsunoda), the Japan Society for the Promotion of Science (JSPS; Grant-in-Aid for Scientific Research [B]-MEXT KAKENHI Grant \#15K08975 [A-M Park], Grants-in-

Aid for Scientific ResearchKAKENHI, $16 \mathrm{H} 07356$ [I Tsunoda]) and Novartis Pharma Research Grants 2017 (A-M Park, I Tsunoda). This paper is subject to the $\mathrm{NIH}$ Public Access Policy. The authors have no other relevant affiliations or financial involvement with any organization or entity with a financial interest in or financial conflict with the subject matter or materials discussed in the manuscript apart from those disclosed.

No writing assistance was utilized in the production of this manuscript.

\section{Acknowledgments}

We thank Mitsugu Fujita, Fumitaka Sato and Seiichi Omura for valuable discussion, and Namie Sakiyama for excellent technical assistance.
Figure 2. Schematic illustration of two protocols for detecting fecal occult blood by the luminol reaction. Visual detection (A) and chemiluminescence measurement (B). (A) Visual detection: (1) Add a luminol dilute solution (dilute the original solution fivefold with deionized-distilled water $\left[\mathrm{ddH}_{2} \mathrm{O}\right]$ ) to pooled feces in a microtube (left) or a piece of feces taken by a toothpick in a white 96-well plate (right); and (2) observe them in dark field. In the presence of blood, blue-white chemiluminescence is visible. (B) Chemiluminescence measurement: (1) Prepare fecal solutions with $\mathrm{ddH}_{2} \mathrm{O}(1 \mathrm{mg} / \mathrm{ml})$ in a microtube; (2) vortex and centrifuge the tube for 2 min; (3) transfer $5 \mu \mathrm{l}$ of the solution (containing $5 \mu \mathrm{g}$ of feces) in a 96-well plate; (4) add $100 \mu$ l of a luminol dilute solution (diluted 500 -fold with $\mathrm{ddH}_{2} \mathrm{O}$ ) to each well; and (5) measure the chemiluminescence signal by a luminometer immediately. (C) Fecal blood scores based on the two luminol protocols. To calculate the chemiluminescence ratios, it is recommended to store the control fecal solution ( $1 \mathrm{mg} / \mathrm{ml}$ ) derived from normal mice. $\mathrm{Hb}$ concentrations have been estimated by comparing with the results of feces mixed with standard $\mathrm{Hb}$ solutions.

$\mathrm{ddH}_{2} \mathrm{O}$ : Deionized-distilled water; Hb: Hemoglobin.

\section{Open access}

This work is licensed under the Creative Commons Attribution-NonCommercial-

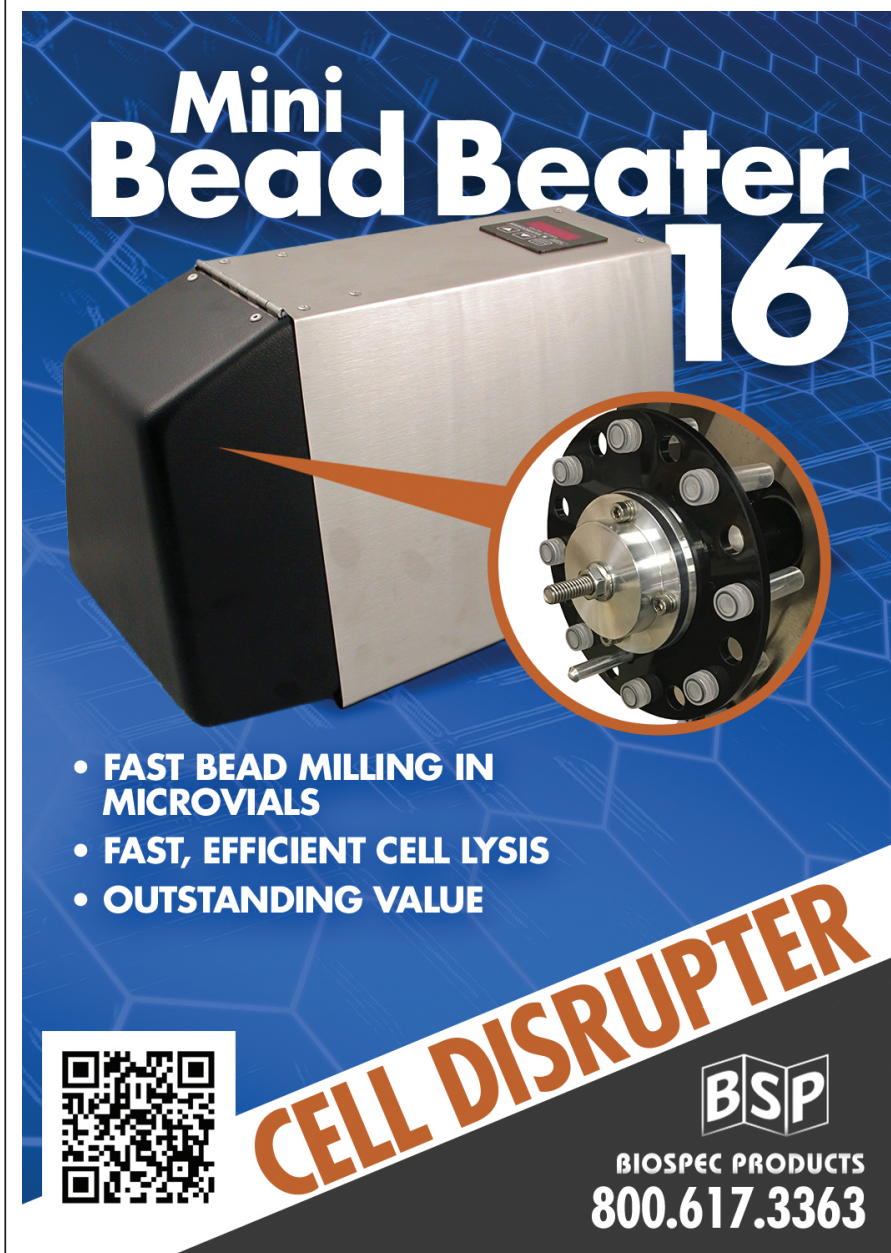


NoDerivs 4.0 License. To view a copy of this license, visit http://creativecommons. org/licenses/by-nc-nd/4.0/

\section{Supplementary data}

To view the supplementary data that accompany this paper please visit the journal website at: www.future-science. com/doi/suppl/10.2144/btn-2018-0017

\section{References}

Papers of special note have been highlighted as:

-• of considerable interest

1. Kobayashi Y, Watabe H, Yamada A et al. Impact of fecal occult blood on obscure gastrointestinal bleeding: observational study. World J. Gastroenterol. 21(1), 326-332 (2015).

2. Federici A, Giorgi Rossi P, Borgia P, Bartolozzi F, Farchi S, Gausticchi G. The immunochemical faecal occult blood test leads to higher compliance than the guaiac for colorectal cancer screening programmes: a cluster randomized controlled trial. J. Med. Screen. 12(2), 83-88 (2005).
3. Levin B, Lieberman DA, McFarland B et al. Screening and surveillance for the early detection of colorectal cancer and adenomatous polyps, 2008: a joint guideline from the American Cancer Society, the US Multi-Society Task Force on Colorectal Cancer, and the American College of Radiology. Gastroenterology 134(5), 1570 1595 (2008).

4. Hewitson P, Glasziou P, Irwig L, Towler B, Watson E. Screening for colorectal cancer using the faecal occult blood test, Hemoccult. Cochrane Database Syst. Rev. (1), CD001216 (2007).

5. Rozen P, Levi Z, Hazazi R et al. Quantitative colonoscopic evaluation of relative efficiencies of an immunochemical faecal occult blood test and a sensitive guaiac test for detecting significant colorectal neoplasms. Aliment. Pharmacol. Ther. 29(4), 450-457 (2009).

6. Stadnicki A, Colman RW. Experimental models of inflammatory bowel disease. Arch. Immunol. Ther. Exp. (Warsz). 51(3), 149-155 (2003).

7. WeberK, Mikulovic V. The luminescence of lumino XI (Yugoslavia). Arhiv. Higijenu rada, Zagreb. (English translated version is available from US Department of the Army, \#AD839542) (1959). -• Introduced the application of the luminol reaction to detect blood spots for judicial and medical purposes.
8. Marquette CA, Blum LJ. Applications of the luminol chemiluminescent reaction in analytical chemistry. Anal. Bioanal. Chem. 385(3), 546554 (2006).

First draft submitted: 19 March 2018; Accepted for publication: 25 July 2018

Address correspondence to: Ah-Mee Park, Department of Microbiology, Kindai University Faculty of Medicine, Osakasayama, Osaka 589-8511 Japan; ampk@med.kindai.ac.jp

\section{To purchase reprints of this article contact:} s.cavana@future-science.com

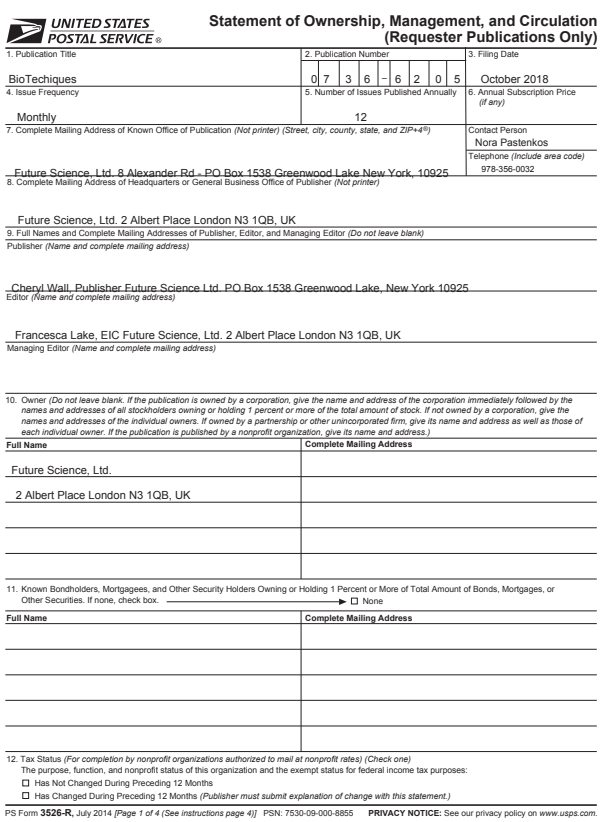

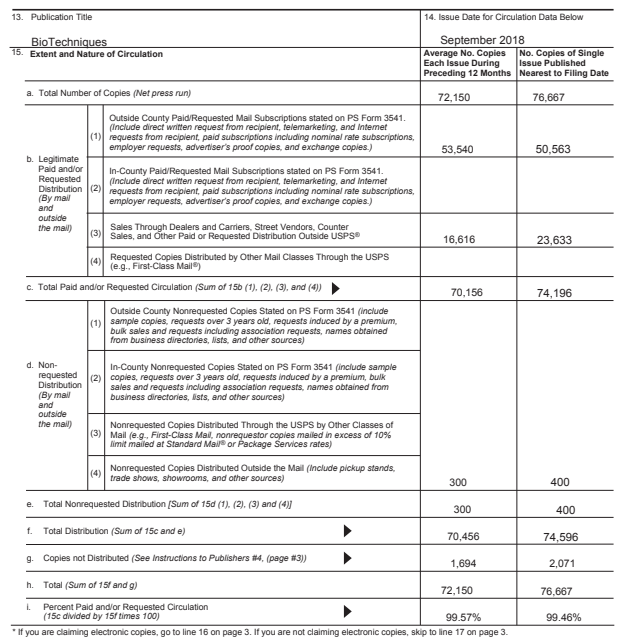

Instructions to Publishers

1. Complete and file one copy of this form with your postmaster annually on or before October

2. In cases where the stockholder or security holder is a trustee in items 10 or 11 , include the name of the person or corporation for whom the trustee is acting. Also include in item 10 the total amount of stock. If not owned by a corporation, give the name and address of each
individual owner. - fi wnede by a partnership or other uninicorporated firm, givivits name and
address as well as the name and address of each individual owner- If the publication is published by a nonprofit organization, give its name and address and complete item 12 . In ittem 11, include all bondholders, mortgagees, and other security holders owning or holding one (1) percent or more of the total amount of bonds, morts
check the box. Use blank sheets if more space is required.

3. Be sure to furnish all circulation information called for in item 15. Free Non-Requested
circulation must be shown in item $15 \mathrm{~d}$.

4. Item $15 \mathrm{~g}$, Copies not Distributed, must include (1) newstand copies returned to the
publisher, (2) estimated returns from news agents, and (3), copies for office use, leftovers pubilied, and all other copies not distributed.
spotes

5. As a requester publication, this Statement of Ownership, Management, and Circulation mus be published, i.e. it it must be printed in an issue that's primary mailed d istribution is produced
not later than Occober 10 for publications issued more frequently than weekky; or not later than October 31 for publications issued weekly or less frequently but more frequently than
monthly; or in the first issue that's primary mailed distribution is produced after October $1 \mathrm{for}$ all other publications

6. In item 16 , check the box if electronic copies are being included in your total distribution and
complete line items 16 a through d.

7. In item 17 , report the date of the issue in which this Statement of Ownership will be published,
if applicable.

It Item 17 must be signed
UNITED STATES
POSTAL SERVICE.

Statement of Ownership, Management, and Circulation
(Requester Publications Only)

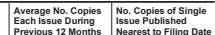

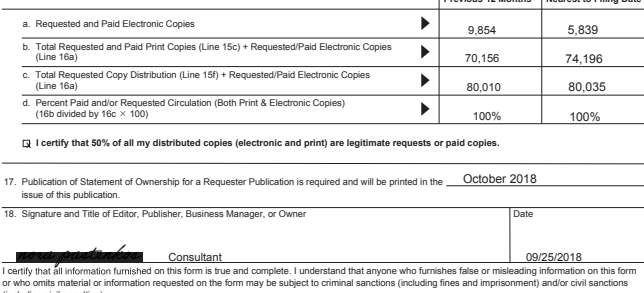

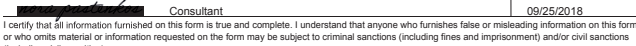

